\title{
Influence of Previous Visual Stimulus or Saccade on Saccadic Reaction Times in Monkey
}

\author{
MICHAEL C. DORRIS, ${ }^{1}$ TRACY L. TAYLOR, ${ }^{2}$ RAYMOND M. KLEIN, ${ }^{2}$ AND DOUGLAS P. MUNOZ ${ }^{1}$ \\ ${ }^{1}$ Medical Research Council Group in Sensory-Motor Neuroscience, Department of Physiology, Queen's University, Kingston, \\ Ontario K7L 3N6; and ${ }^{2}$ Department of Psychology, Dalhousie University, Halifax, Nova Scotia, B3H 4J1, Canada
}

\begin{abstract}
Dorris, Michael C., Tracy L. Taylor, Raymond M. Klein, and Douglas P. Munoz. Influence of previous visual stimulus or saccade on saccadic reaction times in monkey. J. Neurophysiol. 81: 2429-2436, 1999. Saccadic reaction times (SRTs) to suddenly appearing targets are influenced by neural processes that occur before and after target presentation. The majority of previous studies have focused on how posttarget factors, such as target attributes or changes in task complexity, affect SRTs. Studies of pretarget factors have focused on how prior knowledge of the timing or location of the impending target, gathered through cueing or probabilistic information, affects SRTs. Our goal was to investigate additional pretarget factors to determine whether SRTs can also be influenced by the history of saccadic and visual activity even when these factors are spatially unpredictive as to the location of impending saccadic targets. Monkeys were trained on two paradigms. In the saccade-saccade paradigm, monkeys were required to follow a saccadic target that stepped from a central location, to an eccentric location, back to center, and finally to a second eccentric location. The stimulussaccade paradigm was similar, except the central fixation target remained illuminated during presentation of the first eccentric stimulus; the monkey was required to maintain central fixation and to make a saccade to the second eccentric stimulus only on disappearance of the fixation point. In both paradigms, the first eccentric stimulus was presented at the same, opposite, or orthogonal location with respect to the final target location in a given trial. We measured SRTs to the final target under conditions in which all parameters were identical except for the location of the first eccentric stimulus. In the saccade-saccade paradigm, we found that the SRT to the final target was slowest when it was presented opposite to the initial saccadic target, whereas in the stimulus-saccade paradigm the SRT to the final target was slowest when it was presented at the same location as the initial stimulus. In both paradigms, these increases in SRTs were greatest during the shortest intervals between presentation of successive eccentric stimuli, yet these effects remained present for the longest intervals employed in this study. SRTs became faster as the direction and eccentricity of the two successive stimuli became increasingly misaligned from that which produced the maximal SRT slowing in each paradigm. The results of the stimulus-saccade paradigm are similar to the phenomenon of inhibition of return (IOR) in which human subjects are slower to respond to stimuli that are presented at previously cued locations. We interpret these findings in terms of overlapping representations of visuospatial and oculomotor activity in the same neural structures.
\end{abstract}

\section{IN T R O D U C T I O N}

Saccades are rapid eye movements that shift the visual axis from one target of interest in the visual field to another. For the

The costs of publication of this article were defrayed in part by the payment of page charges. The article must therefore be hereby marked "advertisement" in accordance with 18 U.S.C. Section 1734 solely to indicate this fact. vast majority of saccades, the time it takes to initiate a saccade to a suddenly appearing target (saccadic reaction time, SRT) exceeds the minimum afferent and efferent delays calculated for the shortest neural pathway from the retina to the extraocular muscles (Carpenter 1981). A large body of work has shown that as task complexity increases, the duration of neural processing increases, resulting in longer mean RTs (see Coles 1989; McClelland 1979; Meyer et al. 1988 for reviews on mental chronometry). A problem that has received less attention is determining which factors cause changes in SRTs under conditions in which task complexity is held constant.

Most reaction time models assume that a saccade is elicited after a threshold level of activation is surpassed (see Pacut 1977 for review of threshold models). As such, variations in SRTs can be caused by changes in the rate of accumulation of activity toward a constant threshold from trial to trial (Hanes and Schall 1996; McClelland 1979). In addition, variations in SRTs can result when the difference between activity at the time of target presentation and the threshold level is reduced. This can occur either through variations in the threshold level of activity or, more likely, through variations in the baseline level activity from trial to trial (Grice 1968; Nazir and Jacobs 1991). Under most conditions, SRT variations are caused by a combination of these two processes (Carpenter and Williams 1995; Pacut 1977). The neural processing that contributes to SRT, whether by changes in the distance or the rate in which activity accumulates to reach threshold, can be broadly divided into two epochs: pretarget and posttarget.

The most studied of these epochs we will refer to as "posttarget" because these processes occur after target presentation. Posttarget factors that can influence the rate of accumulation of activity toward threshold include target contrast and size (Boch and Fischer 1986), target luminance (Boch and Fischer 1986; Kingstone and Klein 1993; Reulen 1984; Reuter-Lorenz et al. 1991), target eccentricity (Kalesnykas and Hallet 1994), and the number and timing of distracting stimuli (Corneil and Munoz 1996; Schall et al. 1995; see Schall 1995 for review).

Differences in SRTs influenced by the "pretarget" epoch are caused by processes that occur before target presentation (Carpenter and Williams 1995; Dorris and Munoz 1995 1998; Dorris et al. 1997a; Ross and Ross 1980, 1981). Pretarget processes can result in variations in the baseline activity on which the posttarget activity accumulates. If the pretarget baseline activity is high, SRTs will be short. If the baseline activity is low, SRTs will be longer. Pretarget processes allow for the advanced preparation or suppression of movements tailored to task requirements. 
Pretarget processes can be further segregated based on their mechanism of action. A class of pretarget processes reduces SRTs to all target locations due to a general disinhibition of the oculomotor system. This includes reductions in SRTs afforded by variations in the general state of oculomotor readiness (Juttner and Wolf 1992; Paré and Munoz 1996), warning signals (Ross and Ross 1980, 1981; Walter 1964), and fixation disengagement (Dorris and Munoz 1995; Kingstone and Klein 1993; Reuter-Lorenz et al. 1991; Tam and Ono 1994), all of which occur before target presentation. Another class of processes reduces SRTs only to specific target locations by using task-dependent information before target presentation. RTs are reduced when a pretarget cue indicates the likely location of an upcoming target compared with when the cue incorrectly indicates the upcoming target location (manual: Bowman et al. 1993; Jonides and Mack 1984; Posner 1980; saccadic: Abrams and Jonides 1988; Klein and Pontefract 1994). SRTs also covary with the probability of the target being presented at a location within a block of trials (Carpenter and Williams 1995; Dorris and Munoz 1998; Paré and Munoz 1996; Simpson et al. 1997). Another class of pretarget processes impacts SRTs to specific targets through pretarget events that offer no probabilistic information about the location of the upcoming target. The best example of this is the phenomenon known as inhibition of return (IOR) (manual RTs: Maylor 1985; Posner and Cohen 1984; Tanaka and Shimojo 1996; saccadic RTs: Abrams and Dobkin 1994, 1995; Maylor 1985; Rafal et al. 1994; Reuter-Lorenz et al. 1996; Vaughan 1984; see Taylor and Klein 1998 for review of IOR). IOR describes a pattern of results wherein subjects are slower to respond to targets that appear at the same versus a different location as a preceding visual stimulus. Unlike cueing experiments in which the initial stimulus conveys probabilistic information, in IOR, the stimulus is spatially unpredictive about the location of the impending target.

The goal of this study is to examine the influence of this last class of pretarget processes on SRTs in the monkey. In particular, we focus on how two common events (presentation of a visual stimulus and eye movements to a visual stimulus) affect subsequent initiation of saccades. We measured SRTs to a final target during two paradigms in which a previous spatially unpredictive eccentric stimulus is presented to which monkeys are either required to respond with a saccade or to direct no response. The history of prior saccadic movements and visual stimuli impacts the initiation of subsequent saccades in a predictable manner. We account for our observations with a mechanism whereby previous saccadic movements and visual stimuli are coded on a common neural substrate and act to modulate pretarget baseline neural activity to affect subsequent SRTs. This demonstration of IOR in an animal model is the critical first step leading to single-cell recording studies that may uncover the neural mechanisms of this well-studied human phenomenon.

Some of these data have appeared in abstract form (Dorris et al. 1996, 1997b).

\section{METHODS}

\section{Animal preparation}

All procedures were approved by the Queen's University Animal Care Committee and complied with the guidelines of the Canadian
Council on Animal Care. Animals were under the close supervision of the university veterinarian.

Four male rhesus monkeys (Macaca mulatta) weighing between 6 and $8 \mathrm{~kg}$ underwent a single aseptic surgical session to prepare for eye movement and subsequent single-neuron recording (for details see Dorris et al. 1997a; Munoz and Istvan 1998). Eye coils were implanted subconjunctively (Judge et al. 1980) to measure eye position using the magnetic search coil technique (Fuchs and Robinson 1966). Craniotomies were made to allow microelectrodes to access structures in the brain stem for single-cell recordings made after the completion of the behavioral studies described here. Stainless steel screws were threaded into the skull to anchor the acrylic explant that was constructed. The recording chambers, the eye coil leads, and a stainless steel head holder were embedded in the acrylic explant.

At the end of surgery, the animals received a prophylactic injection of antibiotics (penicillin im) for 10 postoperative days. To alleviate any discomfort in the first $2 \mathrm{wk}$ after surgery, the monkeys were also given analgesic medication $(0.01 \mathrm{mg} / \mathrm{kg}$ buprenorphine hydrochloride Buprenex, $5 \mathrm{mg} / \mathrm{kg}$ Flunixin Meglumine, Banamine). Animals were given at least $2 \mathrm{wk}$ to recover from surgery before training began.

\section{Experimental procedures}

Throughout the duration of the experiments, the monkeys were seated in a primate chair with their heads firmly attached to the chair via a head holder. The monkeys faced a tangent screen $86 \mathrm{~cm}$ away that spanned $\pm 35^{\circ}$ of the central visual field. Behavioral paradigms, visual displays, and storage of eye movement data were under the control of a 486 PC computer running a real-time data acquisition system (REX) (Hays et al. 1982). REX controlled the presentation of the targets through D/A converters that moved two mirror galvanometers (General Scanning) in orthogonal planes. These mirrors reflected a light-emitting diode $\left(0.3 \mathrm{~cd} / \mathrm{m}^{2}\right)$ on the translucent screen in front of the monkey while the room was in total darkness. Horizontal and vertical eye and mirror positions were digitized at $500 \mathrm{~Hz}$. All data analysis was performed off-line.

\section{Behavioral paradigms}

Monkeys were trained to perform two behavioral tasks in separate blocks of trials: a saccade-saccade paradigm and a stimulus-saccade paradigm (Fig. 1). Trials were preceded by an intertrial interval (1,000 $\mathrm{ms})$ during which the visual screen was illuminated with diffuse white light $\left(\sim 1.0 \mathrm{~cd} / \mathrm{m}^{2}\right)$ to prevent dark adaptation. The onset of a trial was signaled by the removal of this background light and, after a period of $250 \mathrm{~ms}$, the appearance of the central fixation point (FP). In the saccade-saccade paradigm (Fig. 1A), the monkey was required to look from the central FP to an eccentric target (T1), back to the central FP, and finally to another eccentric target (T2). The details are as follows. Initially, the monkey was required to fixate the central FP for $500 \mathrm{~ms}$ after which it was extinguished and T1 was presented simultaneously. The monkey was required to look at T1 within $500 \mathrm{~ms}$ of its appearance and then maintain fixation on $\mathrm{T} 1$ for $500 \mathrm{~ms}$. T1 was a neutral stimulus in that it did not provide probabilistic information about which of the two possible locations T2 would be presented. T1 was then extinguished, and the central FP was reilluminated. The monkey had $500 \mathrm{~ms}$ to initiate a saccade to the FP. The second period of fixation of the FP had to be maintained for pseudorandomly interleaved periods of 100,500 , or $1,000 \mathrm{~ms}$ before the FP was extinguished. There was a 200-ms "gap" period in which no stimuli was presented followed by the presentation of T2. The total period in which the monkey's eyes remained stationary at the central location of the screen (i.e., both fixation on the FP and during the gap period) was known as the fixation duration (FD; 300, 700, or 1,200 ms; see Fig. 1A). The monkey had $500 \mathrm{~ms}$ to initiate a saccade to $\mathrm{T} 2$ and had to maintain fixation on it for an additional $300 \mathrm{~ms}$.

The stimulus-saccade paradigm (Fig. $1 B$ ) had a similar general 

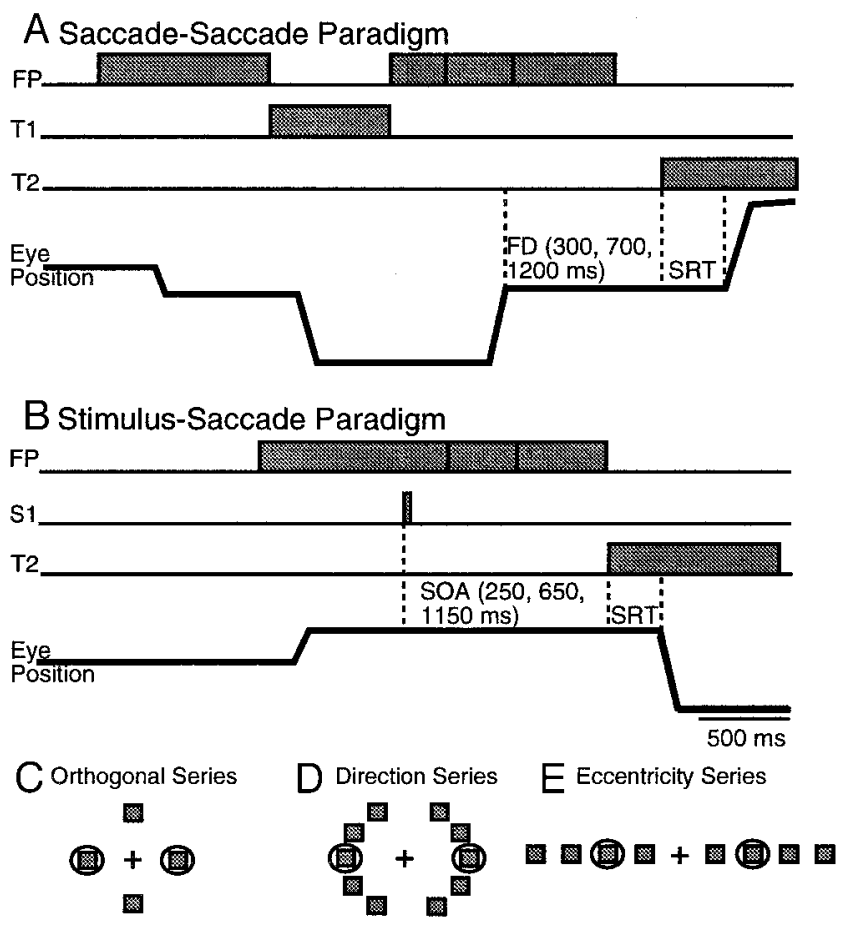

FIG. 1. Schematic of behavioral paradigms. $A$ and $B$ : each horizontal bar represents the presentation of (gray) or possible representation of (white) the fixation point (FP), 1st target (T1), 1st stimulus (S1), or 2nd target (T2). A schematic of horizontal eye position in which up represents rightward movements and down represents leftward movements is also shown. A: saccadesaccade paradigm. $B$ : stimulus-saccade paradigm. $C-E$ : locations of $\mathrm{S} 1$ and $\mathrm{T} 1$ (嘓) relative to the location of the final T2 $(O)$ in the different experiments relative to the position of the central $\mathrm{FP}(+) . C$ : orthogonal series. $D$ : direction series. E: eccentricity series. See METHODS for details.

structure as the saccade-saccade paradigm, but the FP remained visible until the presentation of $\mathrm{T} 2$. The monkey was required to maintain fixation on the central FP and not respond to an spatially unpredictive eccentric stimulus (S1) that was flashed and to later initiate a saccade to T2. S1 was a neutral stimulus in that it did not provide probabilistic information about which of the two possible locations T2 would be presented. The details are as follows. Initially, the monkey was required to fixate the central $\mathrm{FP}$ for $500 \mathrm{~ms}$ before $\mathrm{S} 1$ was flashed for $50 \mathrm{~ms}$. The monkey was required to maintain fixation on the FP and to not respond to $\mathrm{S} 1$. The FP was extinguished after a pseudorandom period of 250,650 , or $1,150 \mathrm{~ms}$ starting from the presentation of S1 [see Fig. $1 B$ : the interval referred to as stimulus onset asynchrony (SOA)]. T2 was presented and the monkey had $500 \mathrm{~ms}$ to initiate a saccade to T2 and had to maintain fixation on it for $300 \mathrm{~ms}$. Even though the SOA and FD intervals were comparable in the two paradigms, it must be noted that the SOA in the saccade-saccade paradigm (i.e., the interval from the beginning of $\mathrm{T} 1$ to the beginning of T2) was much greater than the SOA in the stimulus-saccade paradigm. The time between successive eccentric targets in the former paradigm is necessarily longer to allow time for two intervening saccades (i.e., 1 saccade to T1 and 1 saccade back to the FP; Fig. $1 A$ ).

If the monkey performed a trial correctly, it received a liquid reward. If, however, at any time the monkey did not maintain fixation within the computer-controlled window around the FP or T (usually $3 \times 3^{\circ}$ ), or did not meet the time constraints dictated by each paradigm, the trial was aborted and the monkey did not receive the liquid reward. The monkey worked to satiation, and additional water and fruit were given as necessary.

The location of T1/S1 and T2 were varied systematically, resulting in three target/stimulus configurations for both the saccade-saccade and stimulus-saccade paradigms (Fig. $1, C-E$ ). In the orthogonal series (Fig. 1C), $\mathrm{T} 1$ and $\mathrm{S} 1$ were presented pseudorandomly at $10^{\circ}$ eccentricity either right, up, left, or down (Fig. 1C, 龱) of the central FP (Fig. $1 C,+$ ). T2 was presented pseudorandomly either $10^{\circ}$ to the left or right (Fig. 1C, $\bigcirc$ ) of the central FP. All four monkeys performed the orthogonal series.

In the direction series, the location of T1/S1 was presented pseudorandomly $10^{\circ}$ eccentric to the central FP but with radial directions of $0,30,60,120,150,180,210,240,300$, or $330^{\circ}$ (Fig. $1 D$, 兩). For reference, $0^{\circ}$ direction was to the right of the FP and incremented in a clockwise direction. T2 was then presented pseudorandomly $10^{\circ}$ eccentric either to the right or left side (Fig. 1D, $\bigcirc$ ) of the FP. Two of the four monkeys performed the direction series.

In the eccentricity series, T1/S1 was presented pseudorandomly 5 , 10,15 , or $20^{\circ}$ eccentric on either side of the FP on the horizontal meridian (Fig. 1E, 柶). In blocks of trials, T2 was presented pseudorandomly either 1 ) $5^{\circ} \mathrm{left} / 20^{\circ}$ right, 2) $10^{\circ} \mathrm{left} / 10^{\circ}$ right (Fig. $1 E, \bigcirc$ ), or 3) $20^{\circ}$ left $/ 5^{\circ}$ right of the FP. Only one of the four monkeys performed the eccentricity series.

In both the direction and eccentricity series, the FD and SOA were fixed at 300 and $250 \mathrm{~ms}$, respectively.

\section{Data analysis}

A Sun Sparc2 workstation was used to analyze the data. Computer software determined the beginning and end of each saccade using velocity and acceleration threshold and template matching criteria (Waitzman et al. 1991). These events were verified by an experimenter to ensure accuracy. Trials containing small saccades made during periods of fixation that remained within the computer-controlled windows were eliminated from the analysis.

Throughout the paper, SRTs to T2 (i.e., the time to initiate a saccade after presentation of T2) were considered as a function of the location of $\mathrm{T} 2$ relative to the previous location of T1/S1. In the orthogonal series, orthogonal refers to the collapsed data when the initial T1/S1 was presented $90^{\circ}$ orthogonally (up or down) to the final T2. In all paradigms, "same" refers to when the initial T1/S1 was presented at the same location as the final T2, and "opposite" refers to when the initial T1/S1 was presented on the opposite side of the FP as the final T2. In all cases SRTs were collapsed for the two directions (left and right) of the final saccade.

To test whether the location of the previous T1/S1 affected the SRT to the final T2, a Kruskal-Wallis one-way analysis of variance (ANOVA) on ranks for nonnormal distributions was performed for each condition (3 target configurations: orthogonal, same, and opposite; 3 fixation duration/interstimulus intervals) followed by an all pairwise multiple comparison procedure (Dunn's test at $P<0.05$ significance level). The Dunn's test (a.k.a. Bonferroni $t$-test) is an all pairwise comparison procedure used following nonparametric ANOVAs in which the sample size is different in different groups (Dunn 1961).

\section{R E S U L T S}

\section{Orthogonal series}

SRTs to T2 were influenced by the position of the initial T1 in the saccade-saccade paradigm (Fig. $2 A$ ) or $\mathrm{S} 1$ in the stimulus-saccade paradigm (Fig. $2 B$ ). In the orthogonal series, SRTs were influenced differently in the saccade-saccade and stimulus-saccade paradigms, under similar stimulus orientations. The mean SRTs for the three stimulus configurations (same, opposite, orthogonal) are shown for both paradigms in Fig. 2 for the shortest FD $(300 \mathrm{~ms})$ and SOA $(250 \mathrm{~ms})$. In the saccade-saccade paradigm (Fig. 2A), SRTs were slowest when T1 and T2 were presented at opposite locations (Dunn's method, $P<0.05$ opposite vs. same in 3 of the 4 monkeys; 

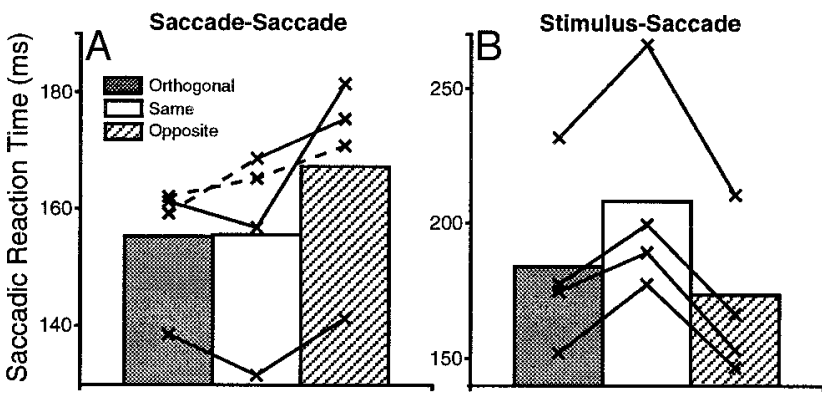

FIG. 2. Saccadic reaction times (SRTs) to T2 in both the saccade-saccade $(A)$ and stimulus-saccade $(B)$ paradigms at the shortest fixation duration (FD) $(300 \mathrm{~ms})$ and stimulus onset asynchrony (SOA; $250 \mathrm{~ms}$ ), respectively. All data are shown collapsed across direction (left and right saccades), and the data for both locations of S1/T1 in the orthogonal condition are also collapsed. Each set of data points $(X)$ connected by lines represent data from 1 monkey, and each bar represents the mean data from all 4 monkeys. Data points connected by solid lines represent a significant difference (Dunn's test $P<0.05$ ) between adjoining data points. Each individual data point consists of between 50 and 200 trials. Note that the scales are different in most of the graphs of this (and the following) figures.

$P<0.05$ opposite vs. orthogonal in 3 of the 4 monkeys). In two monkeys, SRTs were faster when T1 and T2 were presented at the same location in the saccade-saccade paradigm (Dunn's method, $P<0.05$ same vs. orthogonal), but the other two monkeys showed the opposite nonsignificant trend such that there was no difference in the mean data of same versus orthogonal conditions. In contrast, the opposite pattern was observed in the stimulus-saccade paradigm (Fig. 2B). SRTs were the slowest when S1 and T2 were presented at the same location (Dunn's method, $P<0.05$ same vs. opposite in all monkeys; $P<0.05$ same vs. orthogonal in all monkeys). SRTs were the fastest when S1 and T2 were presented in the opposite location in the stimulus-saccade paradigm (Dunn's method, $P<0.05$ opposite vs. orthogonal in 2 monkeys).

The mean difference in SRT for all monkeys between the same and opposite stimulus configurations in the stimulussaccade paradigm was large (34.5 ms). The mean difference in SRT for all monkeys between the opposite and same stimulus configurations in the saccade-saccade paradigm was somewhat reduced $(11.7 \mathrm{~ms})$.

\section{Influence of fixation duration and stimulus onset asynchrony}

We next determined whether the effects of stimulus and saccadic history on final SRT were dependent on the time
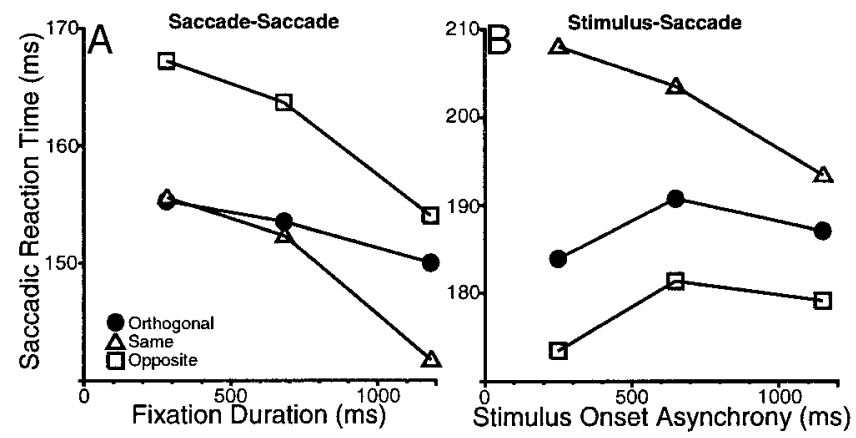

FIG. 3. SRTs to T2 in both the saccade-saccade $(A)$ and stimulus-saccade (B) paradigms as it evolves with FD and SOA, respectively. Each data point represents the mean data from all 4 monkeys in that condition. Each monkey performed between 50 and 200 trials for each data point. between presentation of successive stimuli. In the saccadesaccade paradigm, the time between successive stimuli was influenced by randomly varying FD $(300,700$, and $1,200 \mathrm{~ms}$; Fig. 1A). In this paradigm (Fig. 3A), SRTs decreased in the opposite and same conditions when the FD increased from 300 to $1,200 \mathrm{~ms}$ (Dunn's method, $P<0.05$, in all monkeys), yet the difference between the opposite and same conditions remained with increasing FD (Dunn's method, $P<0.05$ in all monkeys).

In the stimulus-saccade paradigm, the time between the presentation onsets of successive stimuli is defined as the SOA (250, 650, and 1,150 ms; Fig. 1B). In this paradigm (Fig. 3B), the greatest differences in SRT between the same and opposite stimulus configurations were obtained using the shortest intervals between successive stimuli. SRTs decreased in the same condition when the SOA increased from 250 to $1,150 \mathrm{~ms}$ (Dunn's method, $P<0.05$ in all monkeys). The difference between the opposite and same conditions decreased with SOA, although this difference remained in all monkeys at the 1,150 -ms SOA (Dunn's method, $P>0.05$ in 2 of the 4 monkeys).

\section{Influence of spatial disparity between first and second stimulus}

To determine the spatial extent of the influence of the previous stimulus location on the SRT toward T2, we varied independently the direction (Fig. 1D) and eccentricity (Fig. 1E) of T1 and S1.

DIRECTION SERIES. Figure 4 shows that variations in the directional alignment of successive stimuli systematically influenced SRTs when the FD and SOA were fixed at 300 and 250 $\mathrm{ms}$, respectively. The data from two different monkeys and two different T2 locations (Fig. 4, thin lines) are normalized to the longest SRT. The mean data are represented by the squares connected by the thick line.

In the saccade-saccade paradigm (Fig. 4A), the most pronounced increase in SRTs occurred when T1 and T2 were presented on opposite sides of the FP (i.e., $180^{\circ}$ ). The effect diminished with increasing misalignment from opposite. The fastest SRTs occurred when T1 and T2 were presented at the
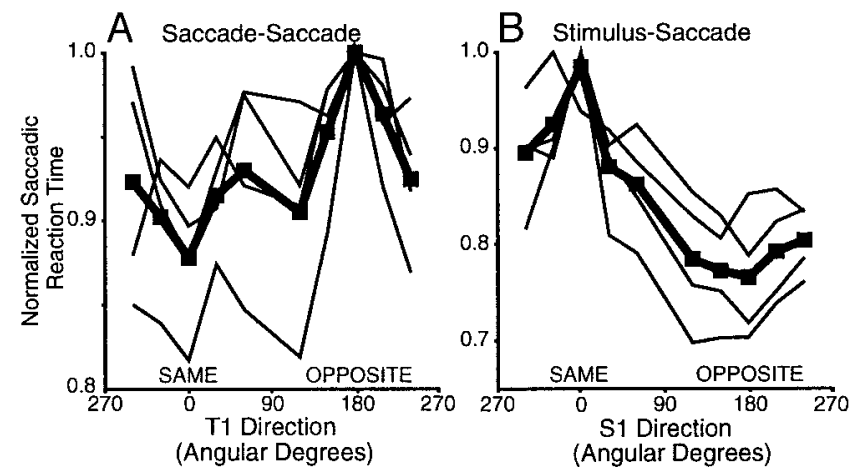

FIG. 4. Effect of $\mathrm{S} 1 / \mathrm{T} 1$ direction on SRT to T2 in the saccade-saccade $(A)$ and stimulus-saccade $(B)$ paradigms. In each panel, the 4 thin lines represent SRT normalized to the longest SRT from the 2 T2 directions (left and right) in each monkey. The thick line is the mean of the 4 individual lines. The data obtained from when the final target was presented $10^{\circ}$ to the left was reversed with respect to the 1st stimulus direction so the data could be presented in the same format as the rightward data. Each data point consists of between 30 and 100 trials. 

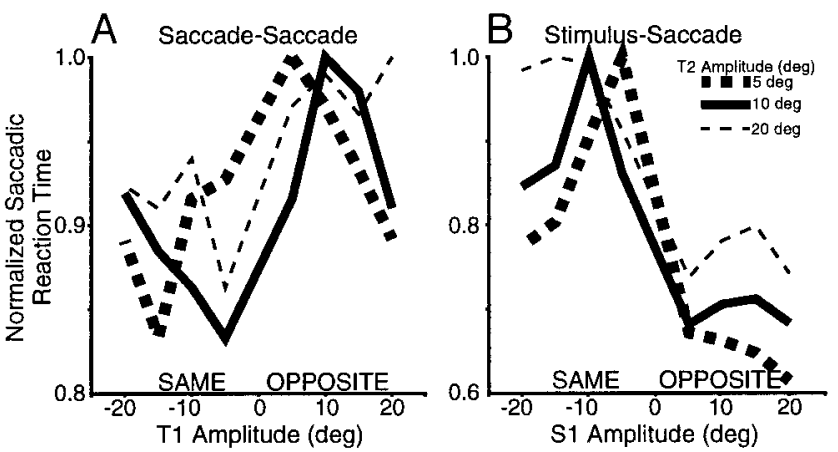

FIG. 5. Effect of S1/T1 eccentricity on SRT to T2 in the saccade-saccade $(A)$ and stimulus-saccade $(B)$ paradigms. Data were obtained from only 1 monkey. Each line represents the SRT normalized to the longest SRT. Data were collapsed across direction (left and right). Each data point consists of between 40 and 80 trials.

same target location and SRTs increased with increasing misalignment of the targets in the same hemifield.

In the stimulus-saccade paradigm (Fig. $4 B$ ), the reverse pattern was observed. In this case, the most pronounced increase in SRT occurred when S1 and T2 were presented at the same location (i.e., $0^{\circ}$ ), and this effect diminished as $\mathrm{S} 1$ and $\mathrm{T} 2$ became increasingly misaligned. The fastest SRTs occurred when S1 and T2 were presented opposite from each other with respect to the FP.

ECCENTRICITY SERIES. Altering the alignment between T1/S1 and $\mathrm{T} 2$ by varying $\mathrm{T} 1 / \mathrm{S} 1$ eccentricity (Fig. $1 E$ ) also affected the final SRT (Fig. 5) in a manner similar to the effects of varying direction. In the saccade-saccade paradigm (Fig. 5A), when T1 and $\mathrm{T} 2$ were presented at equal amplitudes but on opposite sides of the FP, the final saccades had the largest SRTs. As the final saccadic amplitude varied from 5 to 10 to $20^{\circ}$, the amplitude of the previous T1 that elicited the slowest SRT shifted correspondingly toward greater eccentricity (Fig. 5A).

In the stimulus-saccade paradigm (Fig. $5 B$ ), when $\mathrm{S} 1$ and T2 were presented at the same location, it resulted in the slowest SRTs, and as S1 and T2 became increasingly misaligned, the SRTs were reduced.

\section{I S C US S I O N}

The time to initiate a saccade is influenced by the history of previous saccadic eye movements and visual stimuli. In our study, the stimulus configurations that resulted in the largest increase in SRTs were diametrically opposite in the two paradigms. In the saccade-saccade paradigm, when the two successive saccadic targets were presented on opposite sides of the FP, the final SRT was slowest. In contrast, in the stimulussaccade paradigm, when the visual stimulus and saccadic target were presented at the same location, SRTs were slowest. This difference between the same and opposite conditions remained when the time increased between successive stimuli (Fig. 3) but diminished for the stimulus-saccade paradigm. In addition, these interactions diminished when the direction (Fig. 4) and eccentricity (Fig. 5) of the successive stimuli became increasingly misaligned relative to those that produced the maximal effect.

The schematic shown in Fig. 6 illustrates a possible mechanism for our observed results. It depicts a neural structure whose activity represents a topographically organized map of saccade generation and also displays activity in response to the presentation of visual stimuli. Both sensory and motor inputs impinge on the same neurons on this map. It is not until the activity surpasses some threshold level on this map that a saccade is initiated with a vector corresponding to the topographic location of this activity.

We propose that residual inhibition may persist on this map for some time after each saccade or presentation of eccentric stimulus. The onset of the first eccentric stimulus leads to an increase in activity on the map at the locus coding for a saccade to the stimulus location. If the stimulus is the target for a saccade (saccade-saccade paradigm; Fig. 6Ai), the activity will surpass the threshold to elicit the corresponding saccade. If the stimulus is not the target for a saccade but is simply an irrelevant visual stimulus (stimulus-saccade paradigm; Fig. $6 \mathrm{Bi}$ ) the activity will not reach the threshold level necessary to elicit a saccade. In the saccade-saccade paradigm, the activity at the locus coding the saccade must be actively inhibited to
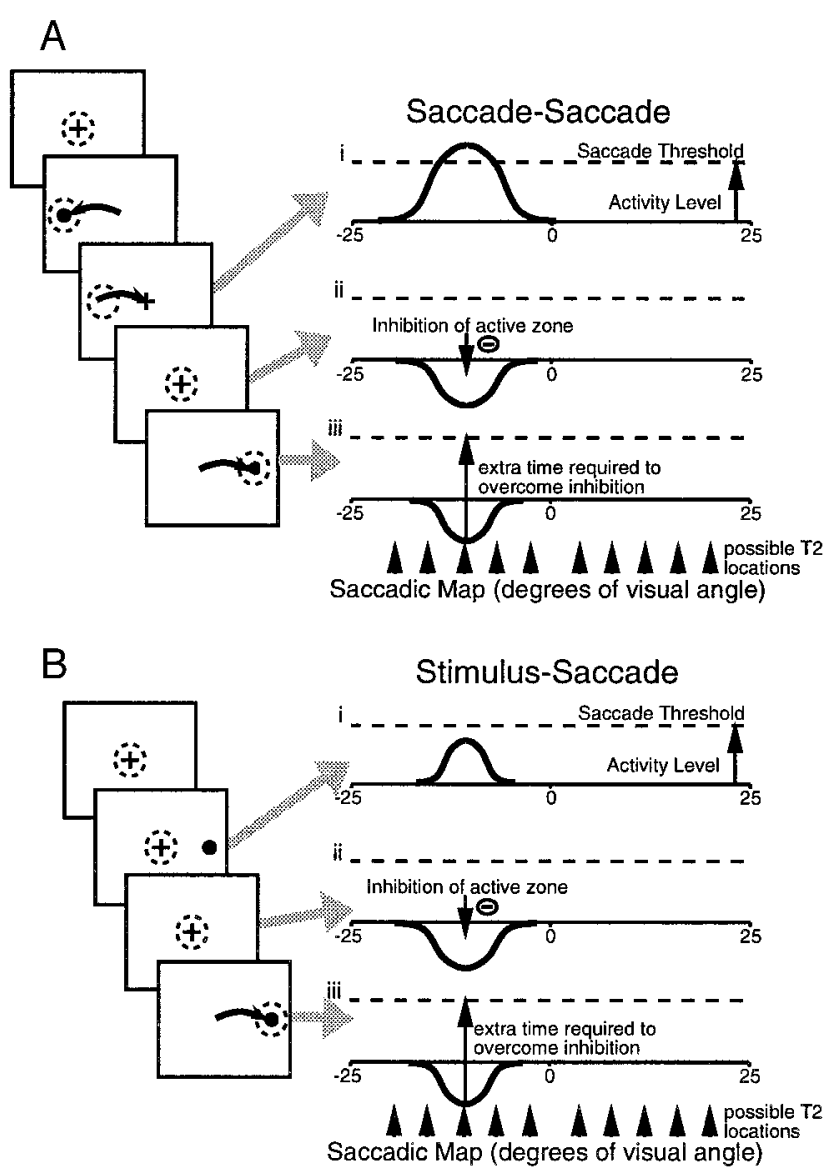

FIG. 6. Schematic of proposed mechanism for pretarget effects on SRT. Panels on the left represent different epochs in the stages of the saccadesaccade $(A)$ and stimulus-saccade $(B)$ paradigms moving from the beginning to end of a trial from top to bottom. Gray arrows point to the corresponding activity of a hypothetical topographic map in which visual stimuli and saccadic movements are coded. Horizontal location is plotted on the $x$-axis and activity level on the $y$-axis. Targets and visual stimuli are presented at either $10^{\circ}$ to the right or left of the central FP and affect activity on the contralateral location on the topographic map. A saccade is elicited only when activity surpasses the saccadic threshold depicted by the horizontal dashed line. The multiple arrows at the bottom of the figures represent possible subsequent target locations that may occur. Depending on the location of a subsequent target, they may fall either within or outside the area of the inhibited active zone resulting from the previous stimulus or saccade, and SRT will be affected accordingly. 
terminate the saccade. For example, the saccade-related burst discharge of saccade-related neurons in the superior colliculus can drop from 500 spikes/s at saccade onset to almost 0 spikes/s by the end of the saccade (Munoz and Wurtz 1995; Waitzman et al. 1991). Because of this presumed active inhibitory process, a valley of inhibition may form at this map location during the intersaccadic interval (Fig. 6Aii). If a saccadic target is presented at or near the location that coincides with this valley of inhibition, extra time will be required to reach threshold, thus resulting in prolonged SRTs (Fig. 6Aiii).

In the stimulus-saccade paradigm (Fig. 6B), the suppression of a saccade to $\mathrm{S} 1$ could result in a reduced level of excitability on a saccadic map (Fig. 6Bii). If so, then extra time would be required to surpass the saccadic threshold when a saccadic target appears (Fig. 6Biii). However, similar paradigms have been shown to not only affect saccadic but also manual RTs, which suggests that this effect may occur in the processing of the input to these motor areas of the brain. The effect of reduced sensory activity (Mangun and Buck 1998; Robinson and Kertzman 1995) would be poorer detection and subsequent slower manual and saccadic RTs through reduced inputs onto both manual and saccadic motor areas.

Although the diametrically opposite stimulus configurations resulted in the slowest responding in the two paradigms, the observed results can be explained if both motor and sensory processes activate a shared neural map coded in oculocentric coordinates like that depicted in Fig. 6. In the saccade-saccade paradigm, if the two last saccades have the same metric, as occurs in the opposite target configuration (from eccentric T1 to center and center to the opposite eccentric T2 location), they will activate the same locus on the map in quick succession. Whereas in the stimulus-saccade paradigm, if $\mathrm{S} 1$ is presented at the same location as $\mathrm{T} 2$, as occurs in the same target configuration, the same locus will also be activated in quick succession, but, in this case, it is caused initially by a sensory mechanism followed by a motor mechanism.

Our finding that SRTs in monkey are slowed as a function of pretarget history of stimuli and saccades is reminiscent of IOR effects that have been studied extensively in humans (for review see Taylor and Klein 1998). IOR is defined as an overall slowing of responses to targets that are presented at the same compared with a different location as a preceding, spatially unpredictive stimulus (Posner et al. 1985). IOR has been forwarded as a mechanism with perceptual and motor components (Abrams and Dobkin 1995), that favors searching for novelty in the visual field (Klein 1988; Posner and Cohen 1984). Once a location has been activated that is of no behavioral interest, subsequent orienting behavior is relatively inhibited from returning to that location. It has been suggested that this safeguards limited resources from being repeatedly squandered at the location of an irrelevant stimulus (Klein 1988; Posner and Cohen 1984).

Although the majority of studies of IOR have elicited it using a peripheral stimulus and measured its effects on manual responses to peripheral targets (see Taylor and Klein 1998), there is strong evidence that activation of the oculomotor system plays a central role in causing IOR (Rafal et al. 1989), and IOR has been shown to delay SRTs (Abrams and Dobkin 1995; Rafal et al. 1994). In considering what may be inhibited by IOR, the human literature converges on perceptual and motor delays that may be tied to slower orienting of attention toward a previously stimulated location (Abrams and Dobkin 1995; Rafal et al. 1994; Reuter-Lorenz et al. 1996; however, see Klein and Taylor 1994). In agreement with a considerable number of studies of IOR in human observers, we have shown that in the monkey, longer SRTs occur when the stimulus and target are presented at the same spatial location in a stimulussaccade paradigm. However, in the few studies employing a similar saccade-saccade paradigm (Rafal et al. 1994; Taylor 1997), humans express IOR when both saccadic targets are presented at the same location, whereas we have shown that in monkeys SRTs are longer when both saccadic targets are presented at opposite locations. To presume that there is a species difference in the saccade-saccade paradigm may be unwarranted for a number of reasons. First, monkeys and humans have not been tested on the same saccade-saccade paradigm, and subtle methodological changes may account for the observed differences. Second, the monkeys may use different strategies due to some form of expectation or due to the overtraining that is required before monkeys can learn the task. If these possibilities are ruled out, there may still exist major neurophysiological differences that will constrain the development of an animal model for research into this phenomenon. Regardless, it is clear that pretarget events can impact SRTs to specific target locations in both species and that these pretarget influences do not depend on the communication of probabilistic information.

The exact neural substrate(s) subserving our postulated mechanism in the monkey is unknown. One of the main criteria of our proposed model (Fig. 6) is shared visuospatial and saccadic activity on the same oculomotor structure. This requirement is ubiquitous in cortical oculomotor regions (see Corbetta 1998; Corbetta et al. 1998 for reviews) including the frontal eye fields (Bruce 1990), supplementary eye fields (Schlag and Schlag-Rey 1987), and the posterior parietal cortex (Andersen 1989) as well as subcortical areas such as the substantia nigra (Hikosaka and Wurtz 1989) and superior colliculus (Sparks and Hartwich-Young 1989). Of these, preliminary data from disparate fields of study point to the superior colliculus as a possible neural structure subserving IOR and the pretarget influences observed in our study. This includes evidence from anatomically based (Rafal et al. 1989; Tanaka and Shimojo 1996), psychophysical (Abrams and Dobkin 1994), neuropsychological (Danziger et al. 1997; Posner et al. 1985), and developmental (Clohessy et al. 1991; Valenza et al. 1994) sources.

The neurons of the intermediate layers of the superior colliculus code both eye movement generation and visual stimuli (see Sparks and Hartwich-Young 1989 for review), and these neurons are organized into an oculocentric map (Robinson 1972). A portion of these neurons have pretarget activity that is related to motor preparation (Dorris and Munoz 1998; Dorris et al. 1997a). This motor preparatory activity is negatively correlated to the SRT of contralateral saccades and positively correlated to the SRT of ipsilateral saccades. This push-pull mechanism by which activity in one region inhibits activity in another region of the superior colliculus (Munoz and Istvan 1998) may provide a mechanism to account for the observed facilitation of SRTs in one direction and the subsequent inhibition of SRTs in the mirror direction observed in the present study (e.g., Fig. 4).

The similarity between our stimulus-saccade results in the 
monkey and IOR reported in humans suggests that we may have an animal model that allows for the application of neurophysiological techniques in tackling the underlying mechanisms of IOR. A comparison of our saccade-saccade and stimulus-saccade findings allow for the possibility that the effects observed in these two paradigms are subserved by the same neural mechanism. We are currently using the results of these experiments to guide single-cell recording studies in the monkey superior colliculus to explicate a neural basis for these phenomena.

We thank A. Lablans, K. Moore, and D. Hamburger for outstanding technical support and I. Armstrong, J. Broughton, B. Corneil, S. Everling, and M. Paré for commenting on an earlier version of the manuscript. This work was supported by a Collaborative Research Grant from the National Sciences and Engineering Research Council (NSERC) of Canada to R. M. Klein and D. P. Munoz. M. C. Dorris was supported by a Queen's University Graduate Fellowship. T. L. Taylor was supported by NSERC and Killam Memorial Trust Fund scholarships. D. P. Munoz is a Medical Research Council of Canada Scientist and Fellow of the EJLB Foundation.

Present address of T. L. Taylor: Vision Research Center, Dept. of Psychology, Vanderbilt University, Nashville, TN 37240

Address reprint requests to D. P. Munoz.

Received 9 November 1998; accepted in final form 3 January 1999.

\section{REFERENCES}

Abrams, R. A. AND Dobkin, R. S. The gap effect and inhibition of return: interactive effects on eye movement latencies. Exp. Brain Res. 98: 483-487, 1994.

ABRAMS, R. A. AND DobKIN, R. S. Inhibition of return: effects of attentional cueing on eye movement latencies. J. Exp. Psych.: HP\&P. 20: 467-477, 1995.

Abrams, R. A. AND Jonides, J. Programming saccadic eye movements. J. Exp. Psychol. Hum. Percept. Perform. 14: 428-443, 1988.

ANDERSEN, R. A. Visual and eye movement functions of the posterior parietal cortex. Annu. Rev. Neurosci. 12: 377-403, 1989.

BOCH, R. AND FISCHER, B. Further observations on the occurrence of expresssaccades in the monkey. Exp. Brain Res. 63: 487-494, 1986.

Bowman, E. M., Brown, V. J., Kertzman, C., Schwarz, U., and Robinson, D. L. Covert orienting of attention in macaques. I. Effects of behavioral context. J. Neurophysiol. 70: 431-443, 1993.

BRUCE, C. J. Integration of sensory and motor signals for saccadic eye movements in the primate frontal eye fields. In: Signals and Sense in Cerebral Cortex. New York: Wiley, 1990, p. 261-314.

CARPENTER, R.H.S. Oculomotor procrastination. In: Eye Movements: Cognition and Visual Perception, edited by D. F. Fischer and R. A. Monty. Hillsdale, NJ: Erlbaum, 1981, p. 237-246.

CARPENTER, R.H.S. AND WILLIAMS, M.L.L. Neural computation of log likelihood in control of saccadic eye movements. Nature 377: 59-62, 1995.

Clohessy, A., Posner, M. I., and Rothbart, M. K. The development of inhibition of return in early infancy. J. Cognit. Neurosci. 3: 346-357, 1991.

CoLES, M.G.H. Modern mind-brain reading: psychophysiology, physiology, and cognition. Psychophysiology 26: 251-269, 1989.

CoRBETTA, M. Frontoparietal cortical networks for directing attention and the eye to visual locations: identical, independent, or overlapping neural systems? Proc. Natl. Acad. Sci. 95: 831-838, 1998.

Corbetta, M., Akbudak, E., Conturo, T. E., Snyder, A. Z., Ollinger, J. M., Drury, H. A., Linenweber, M. R., Petersen, S. E., Raichle, M. E., Van Essen, D. C., AND Shulman, G. L. A common network of functional areas for attention and eye movements. Neuron 21: 761-773, 1998.

CoRNEIL, B. D. AND MuNOZ, D. P. The influence of auditory and visual distractors on human orienting gaze shifts. J. Neurosci. 16: 8193-8207, 1996.

DanZigER, S., FendRICH, R., AND RAFal, R. D. Inhibitory tagging of locations in the blind field of hemianopic patients. Conscious. Cogn. 6: 291-307, 1997.

DoRRIS, M. C. AND MunOZ, D. P. A neural correlate for the gap effect on saccadic reaction times in the monkey. J. Neurophysiol. 73: 2558-2562, 1995.

DorRIS, M. C. AND MunOz, D. P. Saccadic probability influences motor preparation signals and time to saccadic initiation. J. Neurosci. 18: 70157026, 1998

Dorris, M. C., Munoz, D. P., TAylor, T., And Klein, R. Saccadic reaction times are influenced by the metrics of previous saccades in monkey. Soc. Neurosci. Abstr. 22: 2032, 1996.
Dorris, M. C., Paré, M., And Munoz, D. P. Neuronal activity in monkey superior colliculus related to the initiation of saccadic eye movements. J. Neurosci. 17: 8566-8579, 1997a.

Dorris, M. C., Taylor, T., Munoz, D. P., And Klein, R. Saccadic reaction times are influenced similarly by previous saccadic metrics and exogenous cueing in monkey. Cognit. Neurosci. Soc. Abstr. 27, 1997b.

DunN, O. J. Multiple comparisons among means. J. Am. Stat. Assoc. 56: 52-64, 1961.

Fuchs, A. F. AND Robinson, D. A. A method for measuring horizontal and vertical eye movement chronically in the monkey. J. Appl. Physiol. 21: 1068-1070, 1966.

GricE, G. R. Stimulus intensity and response evocation. Psychol. Rev. 75: 359-373, 1968.

Hanes, D. P. AND Schall, J. D. Neural control of voluntary movement initiation. Science 274: 427-430, 1996.

Hays, A. V., Richmond, B. J., AND Optican, L. M. A UNIX-based multiple process system for real-time data acquisition and control. WESCON Conf. Proc. 2: 1-10, 1982

HikosaKa, O. and WuRTZ, R. H. The basal ganglia. In: The Neurobiology of Saccadic Eye Movements, Reviews of Oculomotor Research, edited by R. H. Wurtz and M. E. Goldberg. Amsterdam: Elsevier, 1989, vol. III, p. 257-281. Jonides, J. AND MACK, R. The cost and benefit of cost and benefit. Psychol. Bull. 96: 24-44, 1984.

JudGe, S. J., Richmond, B. J., AND CHU, F. C. Implantation of magnetic search coils for measurement of eye position: an improved method. Vision Res. 20: 535-538, 1980.

JUTTNER, M. AND WOLF, W. Occurrence of human express saccades depends on stimulus uncertainty and stimulus sequence. Exp. Brain Res. 89: 678681, 1992.

Kalesnykas, R. P. And Hallett, P. E. Retinal eccentricity and the latency of eye saccades. Vision Res. 34: 517-531, 1994.

Kingstone, A. And Klein, R. M. What are human express saccades? Percept. Psychophys. 54: 260-273, 1993.

KLEIN, R. M. Inhibitory tagging system facilitates visual search. Nature 334: 430-431, 1988.

Klein, R. M. AND Pontefract, A. Does oculomotor readiness mediate cognitive control of visual attention? Revisited! In: Attention and Performance 15: Conscious and Unconscious Information Processing, edited by C. Umilta and M. Moscovitch. Cambridge, MA: MIT Press, 1994, p. 333-350.

KleIN, R. M. AND TAYLOR, T. L. Categories of cognitive inhibition with reference to attention. In: Inhibitory Mechanisms in Attention, Memory, and Language, edited by D. Dagenbach and T. H. Carr. San Diego, CA: Academic, 1994, p. 113-150.

MANGun, G. R. AND BuCK, L. A. Sustained visual-spatial attention produces costs and benefits in response time and evoked neural activity. Neuropsychologia 36: 189-200, 1998.

MAYLOR, E. Facilitatory and inhibitory components of orienting in visual space. In: Attention and Performance XI, edited by M. I. Posner and O.S.M. Marin. Hillsdale, NJ: Erlbaum, 1985, p. 189-203.

MCClelland, J. L. On the time relations of mental processes: an examination of systems of processes in cascade. Psychol. Rev. 86: 287-324, 1979.

Meyer, D. E., Osman, A. M., Irwin, D. E., AND Yantis, S. Modern mental chronometry. Biol. Psychol. 26: 3-67, 1988.

MunOZ, D. P. AND IstVAN, P. J. Lateral inhibitory interactions in the intermediate layers of the monkey superior colliculus. J. Neurophysiol. 79: 11931209, 1998.

MunOz, D. P. AND WuRTZ, R. H. Saccade-related activity in monkey superior colliculus. I. Characteristics of burst and buildup cells. J. Neurophysiol. 73: 2313-2333, 1995

NAZIR, T. A. AND JACOBS, A. M. The effects of target discriminability and retinal eccentricity on saccade latencies: an analysis in terms of variablecriterion theory. Psychol. Res. 53: 281-289, 1991.

Pacut, A. Some properties of threshold models of reaction latency. Biol. Cybern. 28: 63-72, 1977.

PARÉ, M. AND MunOz, D. P. Saccadic reaction time in the monkey: advanced preparation of oculomotor programs is primarily responsible for express saccade occurrence. J. Neurophysiol. 76: 3666-3681, 1996.

Posner, M. I. Orienting of attention. Q. J. Exp. Psychol. 32: 3-35, 1980.

Posner, M. I. AND CoHEN, Y. Components of visual orienting. In: Attention and Performance $X$, edited by H. Bouma and D. Bouwhuis. London: Erlbaum, 1984, p. 531-556.

Posner, M. I., Rafal, R. D., ChoAte, L. S., AND Vaughan, J. Inhibition of return: neural basis and function. Cognit. Neuropsychol. 2: 211-228, 1985. 
Rafal, R. D., Calabresi, P. A., Brennan, C. W., and Sciolto, T. K. Saccade preparation inhibits reorienting to recently attended locations. J. Exp. Psychol. Hum. Percept. Perform. 15: 673-685, 1989.

Rafal, R. D., Egly, R., AND Rhodes, D. Effects of inhibition of return on voluntary and visually guided saccades. Can. J. Exp. Psychol. 48: 284-300, 1994.

ReULEN, J.P.H. Latency of visually evoked saccadic eye movements. I. Saccadic latency and the facilitation model. Biol. Cybern. 50: 251-262, 1984.

Reuter-Lorenz, P. A., Hughes, H. C., And Fendrich, R. The reduction of saccadic latency by prior offset of the fixation point: an analysis of the gap effect. Percept. Psychophys. 49: 167-175, 1991.

Reuter-Lorenz, P. A., JHA, A. P., And Rosenquist, J. N. What is inhibited in inhibition of return? J. Exp. Psychol. Hum. Percept. Perform. 22: 367-378, 1996.

Robinson, D. A. Eye movements evoked by collicular stimulation in the alert monkey. Vision Res. 12: 1795-1808, 1972.

RoBINSON, D. L. AND KeRTZMAN, C. Covert orienting of attention in Macaques. III. Contributions of the superior colliculus. J. Neurophysiol. 74: 713-721, 1995.

Ross, L. E. AND Ross, S. M. Saccade latency and warning signals: stimulus onset, offset, and change as warning events. Percept. Psychophysiol. 27: 251-257, 1980.

Ross, S. M. AND Ross, L. E. Saccadic latency and warning signals: effects of auditory and visual stimulus onset and offset. Percept. Psychophysiol. 29: 429-437, 1981.

Schall, J. D. Neural basis of saccade target selection. Rev. Neurosci. 6: $63-85,1995$.

Schall, J. D., Hanes, D. P., Thompson, K. G., AND King, D. J. Saccade target selection in frontal eye field of macaque. I. Visual and premovement activation. J. Neurosci. 15: 6905-6918, 1995.
Schlag, J. And Schlag-Rey, M. Evidence for a supplementary eye field. J. Neurophysiol. 57: 179-200, 1987.

Simpson, S., Trappenberg, T., Klein, R. M., and McMullen, P. Saccadic reaction time as a function of target location probability and the gap effect. Cognit. Neurosci. Soc. Abstr. 31, 1997.

Sparks, D. L. AND Hartwich-Young, R. The deep layers of the superior colliculus. In: The Neurobiology of Saccadic Eye Movements, edited by R. H. Wurtz and M. E. Goldberg. Amsterdam: Elsevier, 1989, vol. III, p. 213-255.

TAM, W. J. AND ONo, H. Fixation disengagement and eye-movement latency. Percept. Psychophysiol. 56: 251-260, 1994.

TAnaka, T. and Shimojo, S. Location vs. feature: reaction time reveals dissociation between two visual functions. Vision Res. 36: 2125-2140, 1996.

TAYLOR, T. L. Generating and Measuring Inhibition of Return (PhD thesis). Halifax, Nova Scotia, Canada: Dalhousie University, 1997.

TAYloR, T. L. AND KLEIN, R. M. On the causes and effects of inhibition of return. Psychonomic Bull. Rev. 5: 625-643, 1998.

Valenza, E. L., Simion, F. L., ANd Umilta, C. L. Inhibition of return in newborn infants. Infant Behavior and Development 17: 293-302, 1994.

VAUGHAN, J. Saccades directed at previously attended locations in space. In: Theoretical and Applied Aspects of Eye Movement Research, edited by A. J. Gale and C. W. Johnson. Amsterdam: Elsevier/North Holland, 1984, p. $143-150$.

Waitzman, D. M., Ma, T. P., Optician, L. M., and Wurtz, R. H. Superior colliculus neurons mediate the dynamic characteristics of saccades. J. Neurophysiol. 66: 1716-1737, 1991.

WALTER, W. G. The contingent negative variation: an electrocortical sign of significant association in the human brain. Science 146: 434, 1964. 